\title{
Pirateriebekämpfung vor den Küsten Somalias Expertentreffen zum Thema „Multinational Law Enforcement and Sea Piracy"“*
}

Von Anna Petrig, LL.M., Max-Planck-Institut für ausländisches und internationales Strafrecht, Freiburg*:*

Ende November 2009 trafen sich im Max-Planck-Institut für ausländisches und internationales Strafrecht Experten aus Wissenschaft, Militär, Industrie und Verwaltung, um die Probleme und Herausforderungen zu diskutieren, welche die Missionen zur Pirateriebekämpfung im Golf von Aden mit sich bringen. Im Fokus standen dabei die Fragen, ob das vom UNO-Sicherheitsrat geschaffene Pirateriebekämpfungsregime den Lackmustest des ersten großen multinationalen Polizeieinsatzes bestanden habe und wie mit aufgegriffenen Piraten und ihrer strafrechtlichen Verfolgung zu verfahren sei. Auch ging es um die Frage, ob neue Instrumente zur Bekämpfung der Piraterie am Horn von Afrika notwendig seien.

\section{Polizeimaßnahmen gegen Piraterie und bewaffnete Raubüberfälle auf See}

Alarmiert durch die sprunghafte Zunahme von Piratenangriffen im Golf von Aden hat der UNO-Sicherheitsrat im Dezember 2008 die bestehenden Regelungen gegen Piraterie und bewaffneten Raub auf See wesentlich erweitert. Ende November 2009 hat er die Geltung dieses neuen Pirateriebekämpfungsregimes um ein weiteres Jahr verlängert. Zu unterscheiden sind dabei drei unterschiedliche geographische Bereiche, für die jeweils andere rechtliche Vorgaben gelten, nämlich Taten auf hoher See, solche, die in den somalischen Küstengewässern stattfinden, sowie die Verfolgung der Täter an Land.

Soweit es um Verfolgungsmaßnahmen auf hoher See geht, gilt auch jetzt noch das auf das Jahr 1982 zurückgehende UNO-Seerechtsübereinkommen (SRÜ). Danach dürfen Piratenschiffe aufgebracht, die Besatzung festgenommen und Eigentum an Bord beschlagnahmt werden. Gerade die Beschränkung auf Taten auf hoher See hat in der Praxis aber zu Schwierigkeiten geführt, da Piraten, die sich von

* Der Bericht wurde bereits veröffentlicht in Jusletter, 11. Januar 2010.

** Die Autorin ist Leiterin des Sea Piracy Project des Max-Planck-Instituts für ausländisches und internationales Strafrecht in Freiburg. Zusammen mit Dr. Robin Geiß (LL.M.) hat sie das demnächst erscheinende Buch Counter-Piracy Operations in Somalia and the Gulf of Aden verfasst. Für weitere Informationen zum Sea Piracy Project und Expertentreffen siehe https:// www.mpicc.de/ext/forschung/forschungsarbeit/strafrecht/piracy.htm. 
dort aus in Küstengewässer flüchten sowie solche, die gezielt nur dort angreifen, sich die Lücken dieser rechtlichen Regelungen zunutze machen können.

Aus diesem Grund hat der Sicherheitsrat im Dezember 2008 das bestehende Pirateriebekämpfungsregime bedeutend erweitert. Staaten dürfen die polizeilichen Maßnahmen des SRÜ neu auch in Somalias Küstengewässern ergreifen - eine Handlung, die ohne entsprechende Sicherheitsratsresolution allein dem Küstenstaat Somalia vorbehalten wäre. Da Piraterie per definitionem nur auf hoher See begangen werden kann, wurde weiter der Tatbestand des „bewaffneten Raubüberfalles auf See“ eingeführt, um auch eine Verfolgung von in somalischen Territorialgewässern begangenen Straftaten zu ermöglichen und um zu beschreiben, gegen wen die genannten polizeiliche Maßnahmen zulässig sind. Außerdem ebnete der Sicherheitsrat den Weg für militärische Operationen auf dem somalischen Festland - eine Befugnis, von der bislang kein Gebrauch gemacht wurde. In der Europäischen Union wird zurzeit jedoch die Möglichkeit diskutiert, Militärs der Afrikanischen Union zur Bekämpfung der Piraterie an Land auszubilden.

\section{Grenzen der Polizeibefugnisse}

Die Teilnehmer am Expertentreffen Multinational Law Enforcement and Sea Piracy waren sich weitgehend einig, dass die durch den Sicherheitsrat verliehenen Befugnisse zum polizeilichen Vorgehen gegen Piraten vor den Küsten Somalias als ausreichend erachtet werden können. Kritisiert wurde jedoch, dass die Schranken dieser zum Teil weitgehenden und einschneidenden polizeilichen Befugnisse und Zwangsmaßnahmen gegen Piraten nicht klar umschrieben werden. In den einschlägigen Piraterie-Resolutionen des Weltsicherheitsrates bleibt es nämlich bei einem generellen Verweis auf die anwendbaren Menschenrechte. Da die an den Pirateriebekämpfungsmissionen teilnehmenden Staaten jedoch extraterritorial sowie teilweise in multinationalen Verbänden sowie in einem maritimen Umfeld handeln, ist es höchst umstritten, ob und welche Menschenrechte im vorliegenden Kontext anwendbar sind.

\section{Identifikation von Piraterieschiffen als operationelle Herausforderung}

In den Beiträgen von Vertretern der NATO Operation Ocean Shield, der EU Mission Atalanta sowie von INTERPOL am Expertentreffen kristallisierte sich heraus, dass sich bei der polizeilichen Verfolgung von Piraten trotz genügenden rechtlichen Rahmens eine Reihe praktischer Probleme ergeben. Einhellig wurde namentlich von der Schwierigkeit berichtet, Piratenschiffe als solche zu identifizieren. Diese würden sich kaum von Fischer- oder Schmugglerbooten unterscheiden. Der Umstand, dass Waffen an Bord gesichtet würden, sei nicht sehr aussagekräftig, da aufgrund der prekären Sicherheitslage in dieser Weltregion beinahe alle Schiffe Waffen zu Selbstverteidigungszwecken mitführen. 
Die erfolgreiche Identifikation und die Aufbringung von Piratenschiffen sind oft nur möglich dank intensivem Informationsaustausch und arbeitsteiligem Vorgehen zwischen den verschiedenen Pirateriebekämpfungsmissionen im Golf von Aden. Die bisher beispiellose Anzahl und Art der beteiligten Akteure an einem internationalen Polizeieinsatz - reichend von internationalen Organisationen bis hin zu non-aligned States - bedingte die Schaffung von Kommunikations- und Koordinationsmechanismen. Hierzu wurde im Dezember 2008 die SHADE Group ins Leben gerufen, welche als Forum zum informellen Austausch und zur Erarbeitung von best practices dient und der inzwischen mehr als 25 Staaten sowie Vertreter der EU NAVFOR, der NATO, der Combined Maritime Forces sowie der Schifffahrtindustrie beigetreten sind. Die Schaffung des militärischen Kommunikationssystems Mercury erlaubt den SHADE Group Mitgliedern zudem den Informationsaustausch in Echtzeit. Gemäß den in den Pirateriebekämpfungsmissionen involvierten Experten könnten diese innovativen und effizienten Kommunikations- und Koordinationsinstrumente für künftige multinationale Polizeieinsätze Schule machen.

\section{Untersuchungsphase - Defizite der Beweiserlangung}

Eine weitere große operationelle Herausforderung stellt die Phase der Untersuchung dar, welche sich an die erfolgreiche Identifikation bzw. Aufbringung eines Piratenschiffes und der Festnahme seiner Besatzung anschließt. Es handelt sich um jenen (zum Teil kurzen) Moment, in dem die notwendigen Beweise und Informationen für ein allfälliges einzuleitendes Strafverfahren gesichert und zusammengetragen werden. Hier wird die Schizophrenie des Pirateriebekämpfungsregimes nämlich der Einsatz von militärischen Mitteln und Militärpersonal für eine genuin polizeiliche Aufgabe - besonders spürbar. An Bord von Militärschiffen fehle es schlicht an Wissen und Erfahrung im Bereich polizeilicher Arbeit und darüber, wie eine erfolgreiche strafrechtliche Untersuchung eingeleitet wird. Beweise würden oft nur sehr mangelhaft aufgenommen, wenn nicht sogar - im wörtlichen Sinne über Bord geworfen, wie es wiederholt mit beispielsweise Waffen geschehen ist. Das Nichtaufnehmen oder gar Vernichten von relevantem Beweismaterial würde eine strafrechtliche Anklage teilweise unmöglich machen beziehungsweise später zu Freisprüchen führen.

Um dieses Manko an polizeilicher Fachkompetenz an Bord von Militärschiffen im Einsatz gegen Piraterie zu kompensieren, wurde unter den Experten die Möglichkeit diskutiert, die Militärpolizei und Angehörige der Militärjustiz stärker einzubinden. Es wurde jedoch hervorgehoben, dass diese - obwohl polizeilich ausgebildet - oft ebenfalls keine Kenntnisse über das relevante Strafverfahrensbzw. Beweisrecht hätten, da Piratenprozesse meist in Drittstaaten wie Kenia und nicht im aufbringenden Staat durchgeführt werden. Diese Kenntnis könnte jedoch durch sogenannte shiprider aus Regionalstaaten mit an Bord gebracht werden. Bei shipridern handelt es sich um Sicherheitskräfte (beispielsweise Polizisten) aus 
Drittstaaten, die an Bord von Militärschiffen gebracht und dort anstelle der Militärs des Flaggenstaates Zwangsmaßnahmen ergreifen und sonstige hoheitliche Handlungen vornehmen. Würden diese auch eingesetzt, um Verdächtige festzunehmen, hätte dies zudem den Effekt, dass sich diese Personen bereits in der Gerichtsbarkeit des entsprechenden Regionalstaates befinden, was eine anschließende Überstellung an den Regionalstaat überflüssig machen würde. Als Alternative zu shipridern könnten auch INTERPOLS incident response teams den verschiedenen militärischen Operationen Unterstützung in der Spuren- und Beweissicherung, d.h. in der spezifisch polizeilichen Arbeit, bieten. Schließlich können aber bereits im Rahmen eines rein militärischen Einsatzes und ohne Zurückgreifen auf Militärpolizei bzw. Militärjustiz, shiprider oder INTERPOL die Prozesse zur Erhebung von Beweisen und Sicherung von Spuren optimiert werden, indem entsprechende gesetzlich vorgeschriebene polizeiliche Abläufe in die militärischen Rules of Engagement "übersetzt" werden. Auch die Arbeit der UN Contact Group on Piracy off the Coast of Somalia an einer Vorlage zur Erhebung von Beweisen gemäß kenianischem Beweisrecht reiht sich in Optimierungsbestrebungen dieser Art ein.

\section{Die Rolle privater Sicherheitsfirmen in der Pirateriebekämpfung}

Nicht nur staatliche Akteure, sondern auch private Sicherheits- und Militärfirmen (PMSCs) spielen eine Rolle in der Gewährleistung von Sicherheit in den von Piraterie betroffenen Gewässern. Schiffe, welche bewaffnetes privates Sicherheitspersonal mit an Bord führen, würden weitgehend vor Übergriffen verschont, da sich Piraten sogenannte soft targets aussuchen. Gemäß anwesenden Experten werde der Einsatz von PMSCs in der Pirateriebekämpfung in Zukunft zunehmen, da diese im Vergleich zu staatlichen Streitkräften nicht nur über angemessenere Mittel hierzu verfügen würden - ein Militärschiff sei ein zu träges Instrument, um agile Piraten zu jagen - sondern diese auch schneller den Strategien und Mitteln der Piraten anpassen könnten. Gleichzeitig sei der Einsatz von PMSCs aber rechtlich nicht hinreichend abgesichert. Die wenigen spezifischen Regeln betreffend private Militär- und Sicherheitsfirmen, die in den letzten Jahren entwickelt wurden namentlich die Montreux Good Practices - stellten auf militärische Einsätze in bewaffneten Konflikten oder Besatzungen und nicht auf Polizeieinsätze auf See ab. Namentlich sei nicht klar, ob der Kapitän des zu beschützenden Schiffes oder der private Sicherheitsdienstleister Befehlsgewalt bzw. ultimative Entscheidungskompetenz über Fragen wie das Vorliegen einer Selbstverteidigungssituation oder das Ausmaß der Anwendung von Gewalt habe. Faktisch habe zudem weder der Flaggenstaat noch ein anderer Staat Interesse daran, Regelverstöße durch PMSCs geltend zu machen und das Erstellen des Sachverhalts - beispielsweise die unrechtmäßige Tötung eines Piraten - sei im maritimen Kontext noch weit schwieriger als an Land. Etwas zynisch wurde die Problematik mit der Frage „Wer würde schon nach einem toten Piraten tauchen?" verbildlicht. 


\section{Strafrechtliche Verfolgung von Piraten}

Eine weitere Erkenntnis, welche am Expertentreffen zu Multinational Law Enforcement and Sea Piracy gewonnen werden konnte, ist, dass ein Bruch zwischen der polizeilichen und der strafrechtlichen Verfolgung von Piraten besteht. Während das Zwangsmaßnahmenregime zum Aufbringen von Piratenschiffen und der Festnahme von Verdächtigen sowohl auf rechtlicher Ebene umfassend geregelt scheint als auch auf operationeller Ebene ein wohl beispielloses internationales Engagement zeitigt, sind nur sehr wenige Staaten bereit oder in der Lage, festgenommene Piraten strafrechtlich zu verfolgen. Bislang wurden einzig in den USA, in Frankreich, in den Niederlanden sowie in Spanien Strafverfahren gegen Piraten eröffnet. Mehrheitlich wurden aufgegriffene Piraten an Staaten in der Region vorab an Kenia - zwecks Aburteilung übergeben, obwohl diese meist keinen wesentlichen Bezug zu Täter oder Tat aufweisen. Strafprozesse vorab in Staaten wie Kenia durchzuführen, gebe nicht nur auf rechtlicher Ebene zahlreiche ungelöste Probleme auf, sondern entspreche auch nicht dem von der UN Contact Group on Piracy Off the Coast of Somalia propagierten Gedanken der gleichmäßigen Lastenverteilung in der Pirateriebekämpfung.

\section{Beurteilung der kenianischen Zuständigkeit für Piratenprozesse}

Anlässlich des Expertentreffens warf ein kenianischer Rechtsprofessor ein Schlaglicht auf Probleme und Herausforderungen im Zusammenhang mit der strafrechtlichen Verfolgung von Piraten vor kenianischen Gerichten. Er wies namentlich darauf hin, dass angesichts der unklaren Pirateriedefinition im kenianischen Strafrecht mit dem Merchant Shipping Act im Jahre 2009 ein neuer Straftatbestand eingeführt wurde. Dabei wurde die alte Piraterienorm außer Kraft gesetzt, obwohl die Anklage in bereits anhängigen Verfahren darauf basiert. Eine Verurteilung scheine damit beinahe unmöglich, denn eine solche könne weder auf die nicht länger existierende alte Pirateriestrafnorm noch (wegen des verfassungsrechtlich garantierten Rückwirkungsverbots) auf die zur Zeit der Tat noch nicht in Kraft gewesene neue Pirateriebestimmung gestützt werden. Vorteilhaft für die Strafverteidiger der Piraten dürfte weiter sein, dass das kenianische Pirateriestrafrecht konfligierende Aussagen zur Zuständigkeit der Gerichte enthält. Während der Judicature Act den High Court als zuständig bezeichnet, sieht die Strafprozessordnung eine alternative Zuständigkeit von High Court oder Magistrate Court vor. Angesichts der Tatsache, dass ein Urteil bei fehlender Zuständigkeit des Gerichts nichtig ist, drängt sich eine Klärung der Zuständigkeitsfrage auf.

Auch auf der Ebene des Beweises stößt das kenianische System im Zusammenhang mit den Besonderheiten der Piraterieprozesse an gewisse Grenzen. So können Zeugen nur unmittelbar vor dem Gericht selbst - das heißt weder schriftlich noch per video link-aussagen. Während Angehörige der Streitkräfte zu einer Teilnahme an einem Strafverfahren im Rahmen ihres Dienstverhältnisses verpflichtet werden 
können, trifft dies für Besatzungsmitglieder auf Handelsschiffen nicht zu. Letztere, die vor allem aus den Philippinen stammen, sind teilweise nicht willens, vor kenianischen Gerichten zu erscheinen, da ein solches Unterfangen so zeitintensiv ist, dass es nicht zwischen zwei (oft ein halbes Jahr dauernde) Schiffspassagen erledigt werden kann. Verpasst ein Matrose eine solche Passage, hat dies für ihn einen beträchtlichen Lohnausfall zur Folge.

In der kenianischen Öffentlichkeit dürfte der Umstand, dass die Piratenprozesse eine zusätzliche Bürde für das an sich schon überlastete Justizsystem darstellen, für Unmut sorgen. Diese Wahrnehmung wird wohl auch dadurch verstärkt, dass Piraten unabhängig von der Belastung der Gerichte prioritär abgeurteilt werden. Weiter sieht das Übereinkommen zwischen der EU und Kenia zur Übernahme von Festgenommenen zwecks strafrechtlicher Verfolgung keine Entschädigungsklausel für den Fall vor, dass Kenia Schadenersatz für unrechtmäßigen Freiheitsentzug leisten muss, wenn Angeschuldigte freigesprochen werden sollten. Dies trägt zur Einschätzung bei, dass Kenia im Vergleich zu anderen Staaten eine ungleich große Verantwortung und Last in der Bewältigung des Piraterieproblems im Golf von Aden zukommt.

\section{Internationales Piratentribunal}

Lebendige Diskussionen unter den Experten rief die Frage hervor, ob ein internationales oder internationalisiertes Tribunal die Lösung für das Problem der strafrechtlichen Verfolgung von Piraten sein könnte. Die Idee, ein solches Tribunal analog den ad hoc-Tribunalen für Ex-Jugoslawien oder Ruanda - auf Kapitel VII der UNO-Charta zu stützen, wurde kritisiert, da Piraterie an sich kaum eine Bedrohung des Weltfriedens oder der internationalen Sicherheit darstellt, wie es solche Maßnahmen erfordern. Zudem lässt sich die Schwere des Verbrechens der Piraterie in keiner Weise mit Kriegsverbrechen, Verbrechen gegen die Menschlichkeit oder Völkermord vergleichen. Alternativ wurde die Möglichkeit in Erwägung gezogen, ein Piratentribunal in Form einer spezialisierten Kammer dem Internationalen Seegerichtshof anzugliedern, was einer Ergänzung des Seerechtsübereinkommens bedürfte. Dies wäre nicht nur zeitintensiv, sondern könnte auch daran scheitern, dass Staaten nicht bereit sind, diesen Vertrag, der weit wichtigere Aspekte als Seepiraterie regelt, anzutasten. Am meisten Erfolg dürfte somit wohl das Modell haben, wie es die UN Contact Group on Piracy off the Coast of Somalia vorschlägt, nämlich die Schaffung von spezialisierten Piratenkammern an nationalen Gerichten. Diese Spezialkammern könnten internationale Elemente aufweisen, reichend von finanzieller Unterstützung bis hin zu internationalen Justizbeamten. Aber selbst bei einem internationalen oder internationalisierten Tribunal würde man spätestens bei der Vollstreckung der Sanktionen wieder auf ein rein nationales System zurückgreifen müssen. Ob die Bereitschaft der Staaten, Freiheitsstrafen zu vollstrecken, größer ist als diejenige, strafrechtliche Verfolgung von Piraten durchzuführen, scheint jedoch zweifelhaft. 


\section{Erfolgreiche Bekämpfung der Piraterie?}

Auf dem Expertentreffen herrschte Einigkeit, dass es unmöglich sei, das vom Sicherheitsrat proklamierte Ziel der full eradication of piracy zu erreichen. Wie ein Experte bemerkte, dürfte die vollständige Beseitigung von Piraterie, welche im Bereich der organisierten Kriminalität anzusiedeln ist und zudem vor den Küsten eines failed State stattfindet, wohl ein etwas hoch gegriffenes Ziel sein, wenn man in westlichen Rechtstaaten nicht einmal der Kleinkriminalität Herr wird. Einig war man sich unter den Experten zudem, dass Piraterie kaum alleine mit Mitteln des Polizei- und Strafrechts besiegt werden könne. Möchte man die Gewaltanwendung auf See nachhaltig eindämmen, müssten vielmehr die strukturellen Probleme Somalias gelöst, sprich der bewaffnete Konflikt befriedet und funktionierende staatliche Strukturen aufgebaut werden. 